\title{
Starquake Observations Near Their Teenage Years
}

\author{
An observational target for more than a century, researchers have only \\ very recently spotted "starquakes"-periodic fluctuations in brightness \\ that can reveal secrets about a star's interior.
}

By Katherine Wright

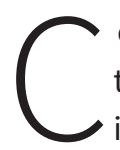

onny Aerts knew she was playing

the long game when, in the 1990s, she started her career in asteroseismology - a field that aims to understand the internal structure of stars through variations in their luminosity. At the time, the phenomenon was mainly explorable theoretically, but that changed dramatically in 2009 when NASA's Kepler mission launched. Designed to find exoplanets, the spacecraft could also collect the stellar brightness data that Aerts, who works at KU Leuven in Belgium, and others had long sought to study. In a paper published today in Reviews of Modern Physics, Aerts outlines the major achievements of the

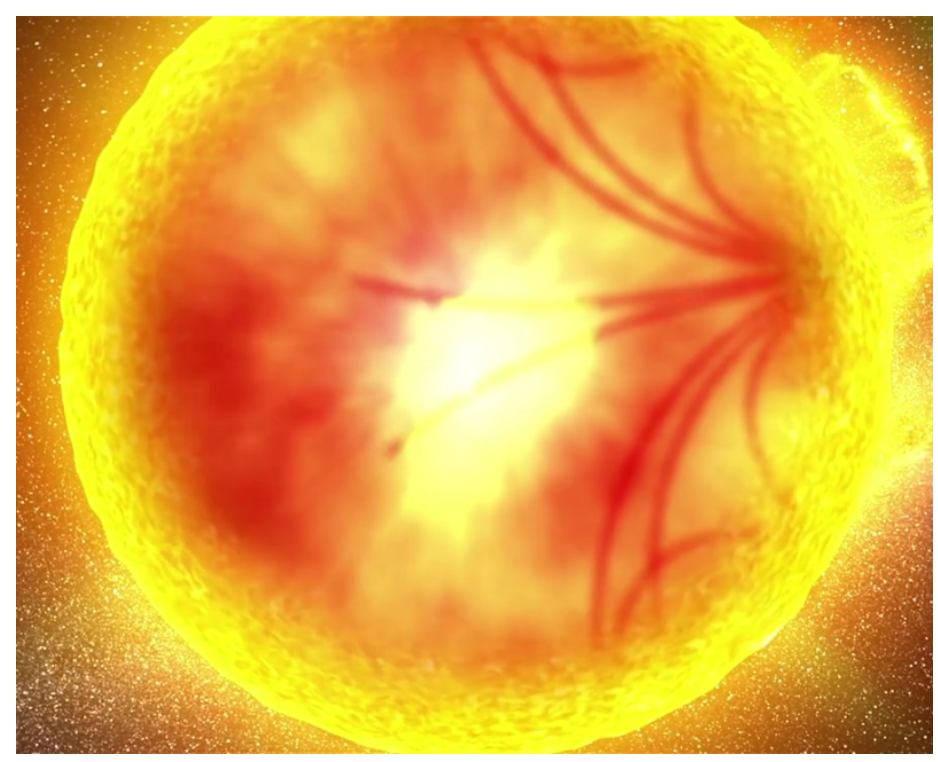

Researchers can use waves traveling through a star to understand the properties of the star's interior.

Credit: Gabriel Perez Diaz/Instituto de Astrofisica de Canarias field's first decade as an observational science and the hopes for its future [1].

Asteroseismologists study stellar oscillations in the form of tiny waves that ripple within a star and across its surface. These wave phenomena-or "good vibrations" as Beach-Boy-fan Aerts calls them-have a variety of sources including the boiling of a star's outer gas layers and the gravitational tugging of nearby planets or stars. The oscillations usually go by the term "starquakes" because of their resemblance to seismic waves that reverberate across the Earth. It is this resemblance that gave Aerts and others the hope that they could use starquakes to understand the swirling plasma inside stars-just as seismiologists use earthquakes to probe our planet's rocky interior. But until Kepler's arrival in 2009, asteroseismologists' plans were foiled either by Earth's atmosphere or by short mission durations.

The waves from a starquake cause the stellar surface to swell and contract, causing its light output to increase or decrease by a tiny amount. Astronomers on Earth are forced to look for those changes through an atmosphere that also fluctuates in its opacity. Those atmospheric fluctuations are thousands of times bigger than the one-part-in-a-million brightness changes caused by starquakes, making starquakes extremely hard to observe using ground-based telescopes. The Kepler telescope-which breached the atmosphere-changed that. "All of a sudden we had new eyes," Aerts says. "We could see what was happening."

During its four-year mission, Kepler tracked the brightness changes of over 200,000 stars. Those observations allowed 
researchers to unravel starquake behaviors with different periods, from the roughly minute-spaced rhythms of Sun-like stars to the day-long cycles of heavier stars. Hundreds of quakes may be rippling through a star at any one time, which, along with other factors, can mean the overall beating pattern of a large star can take years to repeat, Aerts says. "Kepler allowed us to study long timeframes. We could not do that by any other means."

The data have thrown up surprises, the main one being that the theories of stellar rotation did not match observations. The general picture was that as a star ages, the outer layers of its gas expand and the inner layers contract, causing the core to rotate faster. The data confirmed this trend-finding faster core rotation in older stars compared to younger ones, but the measured spin-up was 10 to 100 times slower than theories predicted. This mismatch had implications for models of how chemical species mix inside a star and for expectations of a star's lifetime. "We hadn't anticipated that our theory could be so wrong," Aerts says. "For me, finding that problem was the biggest achievement of the field in the last ten years."

The reasons behind the mismatch have yet to be fully understood, but Aerts and others hope that some of the missing pieces will be filled in by other missions, such as NASA's TESS, which is currently orbiting Earth, and the European Space Agency's PLATO, which is expected to launch at the end of 2026. Unlike TESS and Kepler, PLATO is specifically designed to collect starquake data and-for at least two years-will capture the brightness data every 32 seconds (as compared to 30 minutes for Kepler) for tens of thousands of stars, allowing researchers to study quakes in a wider mass range of stars. "Our field has a very bright future," Aerts says. Saskia Hekker, an astrophysicist at the University of Heidelberg and the Heidelberg Institute for Theoretical Studies, both in Germany, agrees. Even without future data, she notes that researchers have barely scratched the surface on the asteroseismology information contained in the Kepler and TESS data. "There is still so much to be learned," she says.

Correction (22 January 2021): The story has been updated to correct the affiliation of Saskia Hekker, which is the University of Heidelberg and the Heidelberg Institute for Theoretical Studies.

Katherine Wright is a Senior Editor for Physics.

\section{REFERENCES}

1. C. Aerts, "Probing the interior physics of stars through asteroseismology," Rev. Mod. Phys. 93, 015001 (2021). 